\title{
On the effect of the non-constant approach velocity on the liquid film drainage
}

\author{
Runci Song ${ }^{1}$, Luchang Han $^{1}$, Ling Zhang ${ }^{1}$, and Shangyu Tang ${ }^{1}$ \\ ${ }^{1}$ Xiangtan University
}

July 14, 2020

\begin{abstract}
A coupling framework for modeling the non-constant-velocity approach of two fluid particles and the curved film drainage was developed, and an improved model was presented to predict the variable-velocity approach. Using this framework, the effect of the constant-velocity and variable-velocity approach on liquid film drainage was investigated. Two film drainage models based on immobile interface and fully mobile interface were adopted. The simulation results showed that the film thinning rate of the former is much less than that of the latter. In the case of constant-velocity approach, the immobile interface model showed a relatively flat curved film, while in the case of variable-velocity approach, three types of film, wimple, pimple and dimple, can be found. The different combinations of the drainage models and the approach velocity boundary conditions were compared with the experiments. The fully mobile interface model with variable-velocity approach can reasonably predict the coalescence and rebound of bubbles.
\end{abstract}

\section{Hosted file}

Manuscript_Filmdrainagemodel.pdf available at https://authorea.com/users/342850/articles/ 469653-on-the-effect-of-the-non-constant-approach-velocity-on-the-liquid-film-drainage 